\title{
Comparação entre um abrigo meteorológico de baixo custo e a estação meteorológica oficial no INMET, em Santa Maria (RS).
}

\author{
Comparison between a low cost meteorological shelter and the official meteorological \\ station of INMET in Santa Maria (RS).
}

\author{
Ismael Luiz Hoppe ${ }^{1}$, Amanda Comassetto Iensse ${ }^{2}$, João Paulo Delapasse Simioni ${ }^{3}$, \\ Cássio Arthur Wollmann ${ }^{4}$ \\ 1, 2, 3 Alunos - Departamento de Geociências, UFSM, \\ ${ }^{4}$ Orientador / Professor - Departamento de Geociências, UFSM
}

\begin{abstract}
Resumo
Este trabalho teve por objetivo comparar um Abrigo Meteorológico de Baixo Custo (ABC) com a estação meteorológica oficial do Instituto Nacional de Meteorologia - INMET, instalado na Universidade Federal de Santa Maria (UFSM), Rio Grande do Sul. O ABC foi instalado na Estação meteorológica da UFSM a aproximadamente 1,5 metros de altura. Apesar de o Datalogger ficar exposto por 43 dias, apenas foram usados os dados dos dias 10/03/2013 a 10/04/2013, durante três horários diários, às 9 h, às 15 h e às 21 h. O pluviômetro que está contido no ABC é feito de PVC e possui um funil com diâmetro diferente, com tampas em ambas as pontas para evitar a evapotranspiração. Com os dados coletados criaram-se os gráficos de temperatura, umidade relativa e ponto de orvalho, tanto do $A B C$ quanto da estação oficial, para os três horários de coleta. Também foi feito o cálculo de um indice de conversão, onde os dados da $A B C$ foram convertidos para os da estação do INMET. Os resultados iniciais apresentam-se suavemente mais dispersos no $A B C$ do que na estação do INMET. As médias de temperatura diferenciam-se por $1,3^{\circ} \mathrm{C}$. No que diz respeito à umidade relativa do ar, a média variou em aproximadamente $8 \%$, sendo $U R_{A M B C}>U R_{I N M E T}$ Calculou-se ainda a relação entre a quantidade de água coletada pelo pluviômetro de PVC e o pluviômetro oficial.
\end{abstract}

Palavras-chave: Abrigo meteorológico de baixo custo. pluviômetro. temperatura. umidade. Santa Maria-RS.

\begin{abstract}
This work concerns in to compare a Low cost meteorological shelter $(A B C)$ with the Official meteorological station of INMET installed at the Federal University of Santa Maria (UFSM), Rio Grande do Sul. The ABC was installed at the INMET station approximately 1,5 meters of height. Although the Datalogger get exposed for 43 days, only the data collected between the days 03/10/13 and 04/10/13 were used, during three different daily schedule, at $9 a m, 3 p m$ and $9 \mathrm{pm}$. The tax of rain, which was attached to the INMET station, is made of PVC and has a funnel with a different diameter and 2 covers each side that prevents evaporation. With the data collected the temperature, relative humidity and dew point graphics were created with the help of the Microsoft Office Excel software. Also it was made a calculation of a conversion index, where the data collected from de $A B C$ station were converted to the INMET station. The initial results presents gently more dispersed in $A B C$ graphic than INMET's. The average temperature has a difference of $1.3^{\circ} \mathrm{C}$. Talking about the air relative humidity, the average varies in approximately $8 \%$, the air relative humidity of $A B C$ is greater than INMET's. It was calculated the ratio between the amount of water collected by the PVC gauge and official one.
\end{abstract}

Keywords: Low Cost Meteorological Shelter. Rain. Temperature. Humidity. Santa Maria - RS. 


\section{Introdução}

É antiga a necessidade do ser humano em mensurar o que acontece na atmosfera do nosso planeta. Para desvendar esses fenômenos são necessários muitos materiais e equipamentos, porém muitas vezes as instituições e departamentos não dispõem de recursos financeiros para esses estudos. Diante da dificuldade de aquisição desses materiais, a criatividade é estimulada para a construção de equipamentos de baixo custo e de fácil manuseio (Monteiro, 1991).

Deste modo, levando em consideração os altos preços dos instrumentos de medições climáticas aliados à baixa destinação de verbas a projetos em institutos e departamentos para a aquisição de instrumentos de análise e medição climáticas, alternativas são postas em práticas, a fim de desenvolver instrumentos e mecanismos artesanais capazes de suprir a demanda requerida pelos estudos climáticos (temporal e/ou sazonal).

A pesquisa em climatologia normalmente depende de uma densidade relativamente alta de observações espaciais e temporais, o que dificulta a aquisição de equipamentos e suprimentos básicos para a operação e funcionamento deles, tais como baterias, painéis solares, microcomputadores, em número suficiente para as necessidades de alguns temas de pesquisa dessa área do conhecimento (Armani e Galvani, 2004).

Com isto, alguns autores, entre eles, Armani e Galvani (op. cit.) desenvolveram um abrigo meteorológico de baixo custo $(\mathrm{ABC})$ com o objetivo de oferecer métodos de análise climática que ofereçam um baixo custo e uma fácil aplicação, apresentando como base instrumentos de ótima aceitação existentes no mercado.

\section{Metodologia}

Para a realização desta pesquisa, primeiramente confeccionou-se o $\mathrm{ABC}$ baseando-se no modelo proposto por Armani; Galvani (2004) e Milanesi; Galvani (2012), e em seguida instalou-o na Estação Meteorológica Oficial do INMET, localizada na Universidade Federal de Santa Maria (Figura 01) à latitude $29^{\circ} 43^{\prime} 29.78^{\prime \prime}$ sul, longitude $53^{\circ} 43^{\prime} 13.65^{\prime \prime}$ oeste e altitude de 89 metros, instalada junto ao Departamento de Fitotecnia, em campo aberto a aproximadamente 1,5 metros de altura.

Os instrumentos permaneceram instalados em campo, junto à Estação Meteorológica da UFSM, da Rede INMET, por 43 dias, coletando dados de temperatura e umidade do ar a cada hora, e o total de precipitação para o período. Apesar do Datalogger permanecer exposto durante 43 dias, foi considerado para a pesquisa dados obtidos em um mês de coleta, mais precisamente entre os dias 10/03/2013 e 10/04/2013, durante três horários diários, às $9 \mathrm{~h}$, às $15 \mathrm{~h}$ e às $21 \mathrm{~h}$.

Após coletados os dados tanto do $\mathrm{ABC}$ quanto da estação do INMET, iniciou-se a confecção os gráficos de temperatura, umidade relativa e ponto de orvalho, para os três horários diários de coleta com a utilização do software Microsoft Office Excel, bem como a soma do total de chuva precipitado no período.

O abrigo meteorológico foi confeccionado com chapas de ferro galvanizado e suas dimensões são $11,5 \mathrm{~cm}$ de altura e $18 \mathrm{~cm}$ de diâmetro, sendo pintado de cor branca; dentro do abrigo foi colocado o Datalogger para fazer a coleta das temperaturas e umidade, configurado para realizar coleta automática às 9,15 e 21 horas; o pluviô-

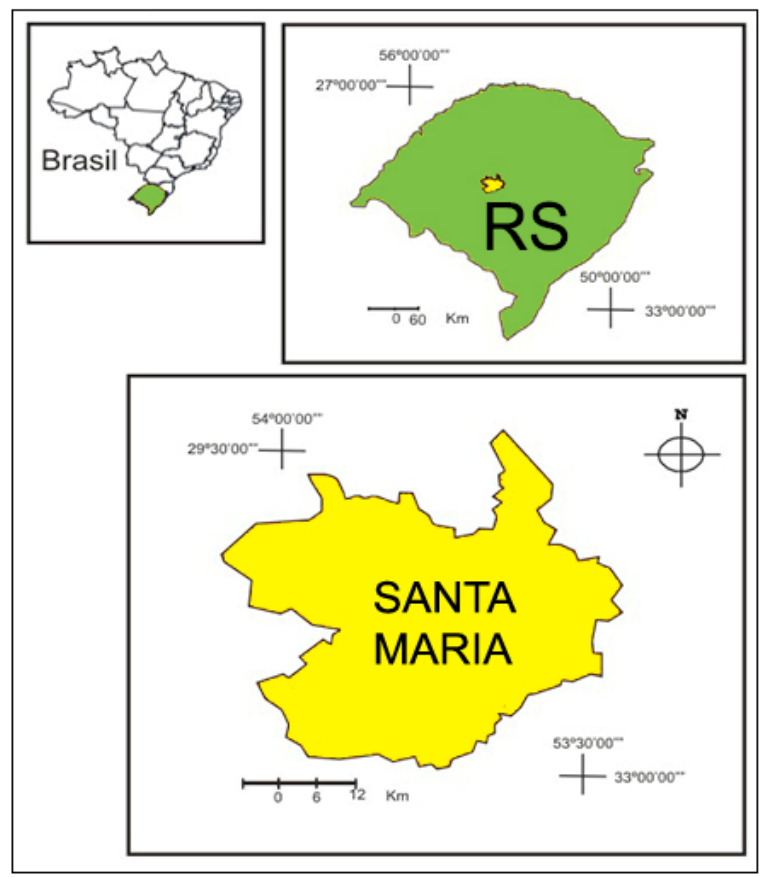

Figura 01 - Localização do município de Santa Maria - RS

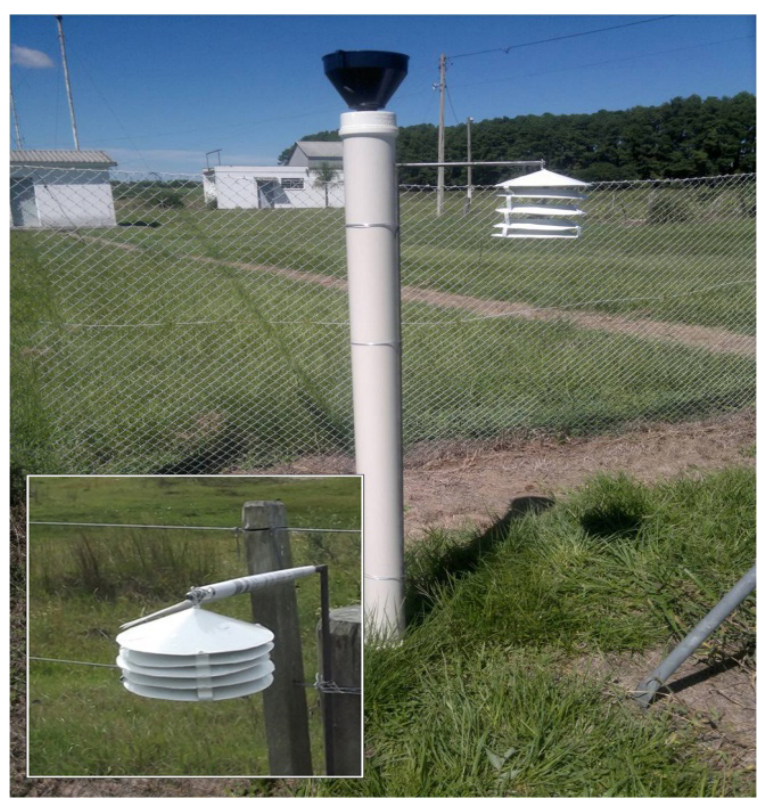

Figura 02 - Abrigo meteorológico e pluviômetro 
metro é feito de cano PVC (100mm), com um funil de $16 \mathrm{~cm}$ de diâmetro instalado sobre a abertura superior. Os equipamentos foram expostos em campo aberto a aproximadamente 1,5 metros de altura, conforme normas da OMM.

Segundo Armani; Galvani (op.cit.), o abrigo de baixo custo (ABC) de ventilação passiva foi inspirado no modelo 41003 10-Plate Gill Radiation Shield da Campbell, utilizado para proteger o sensor Humicap. O ABC permite uma boa ventilação natural por todos os lados, inclusive por baixo, mas não permite a entrada de chuva e de radiação solar direta em nenhum momento do dia, pois há uma área de superposição entre os "anéis" (inferior e superior), que bloqueia a entrada delas (figura 02).

Evidentemente a radiação solar difusa entra no abrigo e interage com o sensor que está dentro dele, assim como acontece no abrigo meteorológico padrão, cujo fundo é totalmente permeável à radiação solar refletida pelo solo, bem como à radiação de onda longa emitida pela superfície.

O custo total para a confecção de um ABC com o Datalogger, por unidade, é de $\mathrm{R} \$ 300,00$, o que equivale a US\$132,74, com a cotação do dólar em (19/11/2013). O preço do abrigo da Campbell (sem Datalogger) é de US\$198,95 (R\$640, 62), sem as taxas aduaneiras.

\section{Análise e Discussão dos Resultados}

Os resultados de Temperatura do Ar, Umidade Relativa e Ponto de Orvalho serão apresentados separadamente a fim de um melhor entendimento e visualização dos resultados obtidos.

\section{1 - Temperatura do Ar}

A partir da tabela 1 verifica-se que os dados relativos à Tabc são suavemente mais dispersos que os dados relativos à TUFSM.

Tabela 1 - Séries de temperatura - parâmetros estatísticos elementares

\begin{tabular}{|c|c|c|c|}
\hline Parâmetros & $\begin{array}{l}\mathrm{T}_{\mathrm{ABC}} \\
\left({ }^{\circ} \mathrm{C}\right)\end{array}$ & $\begin{array}{l}\text { TUFSM } \\
\left({ }^{\circ} \mathrm{C}\right)\end{array}$ & $\begin{array}{c}\text { T }_{\text {ABC-T }}-T_{\text {UFSM }} \\
\left({ }^{\circ} \mathrm{C}\right)\end{array}$ \\
\hline Média & 22,8 & 21,5 & 1,3 \\
\hline Mínimo & 15,6 & 15,8 & $-0,2$ \\
\hline Máximo & 32,7 & 32,4 & 0,3 \\
\hline Amplitude & 17,1 & 16,6 & 0,5 \\
\hline Desvio médio & 3,7 & 3,0 & 0,6 \\
\hline $\begin{array}{l}\text { Desvio } \\
\text { Padrão }\end{array}$ & 4,4 & 3,7 & 0,7 \\
\hline
\end{tabular}

As médias diferenciam-se por 1,3 graus, e a diferença entre as amplitudes é muito pequena, ou seja, apenas um décimo de graus, sendo que a maior amplitude é de TABC. Comparando-se os valores máximos de TABC e Tufsm, nota-se que a diferença é de apenas $0,3^{\circ} \mathrm{C}$ ( TABC $>$ TUFSM). No entanto, em relação aos valores mínimos, TAвс é 0,2 graus menor que TuFsM.

Deste modo confirma-se a afirmação de Armani; Galvani (op. cit.) na qual o ABC, nos horários de maior insolação e aquecimento, tem-se um aumento da temperatura do ar dentro do abrigo decorrente da emissão de ondas longas pela chapa de metal que compõe o abrigo e, por outro lado, nos horários de maior resfriamento, por ser mais ventilado e aberto que TUFSM, diminui a temperatura do ar dentro do abrigo.

Nos gráficos é possível verificar que os dados relativos à TABC são suavemente mais dispersos que os dados relativos à TUFSM. Em relação aos valores mínimos (figura 03), Tabc é $0,2^{\circ} \mathrm{C}$ menor que TufsM.

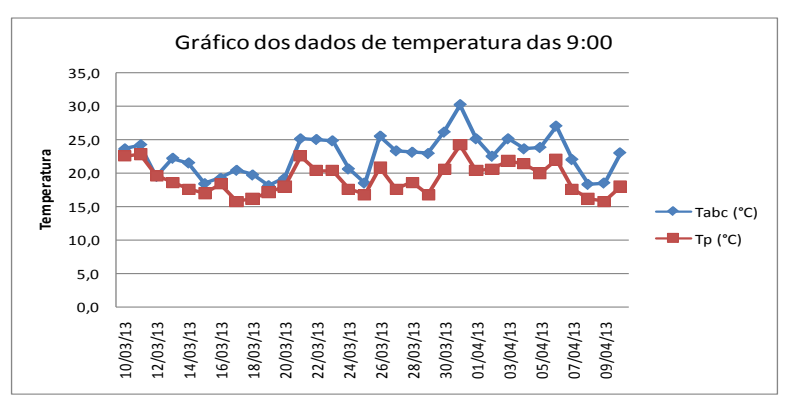

Figura 03 - Gráfico dos Dados de Temperatura das 9 horas

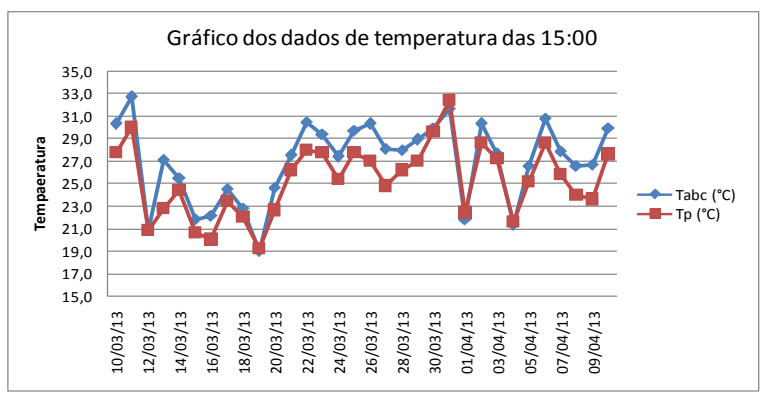

Figura 04 - Gráfico dos Dados de Temperatura das 15 horas

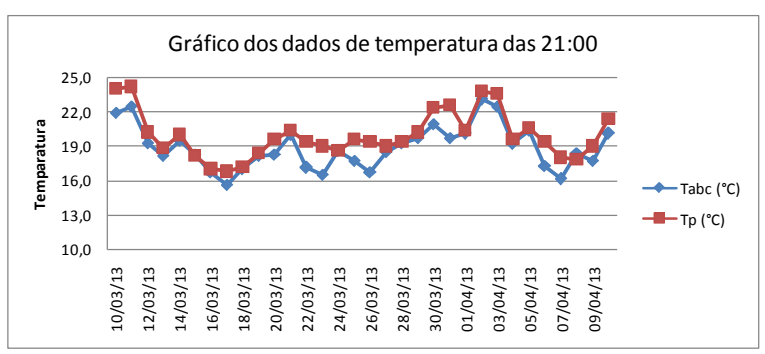

Figura 05 - Gráfico dos Dados de Temperatura das 21 horas 
Comparando-se os valores das $15 \mathrm{~h}$ (figura 04) de Tabc e TuFsm, nota-se que a diferença é de $0,3^{\circ} \mathrm{C}$ ( TABC $>$ TUFSM), ocorrendo o mesmo com os valores das $21 \mathrm{~h}$ (figura 05).

As médias (figura 06) diferenciam-se por 1,3 graus, e a diferença entre as amplitudes é muito pequena, ou seja, apenas um décimo de graus, sendo que a maior amplitude é de ТАBC.

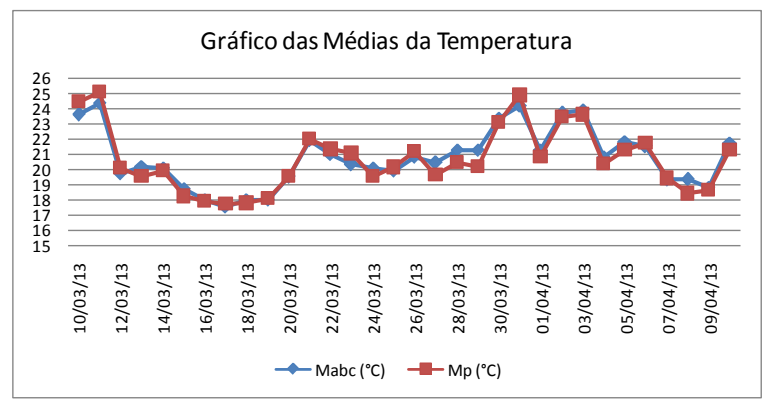

Figura 06 - Gráfico das médias de Temperatura

$\mathrm{O} A B C$, nos horários de maior insolação e aquecimento, tem um aumento da temperatura do ar dentro do abrigo decorrente da emissão de ondas longas pela chapa de metal que compõe o abrigo e, por outro lado, nos horários de maior resfriamento, por ser mais ventilado e aberto que abrigo padrão, diminui a temperatura do ar dentro do abrigo.

\section{2 - Umidade Relativa do Ar}

A partir da tabela 2 pode-se notar que os dados de Uabc são mais dispersos que Up (Estação Oficial), e deste modo nota-se que as variações ocorridas nestes parâmetros estatísticos entre Uabc e Up não estão contidas dentro do erro instrumental de ambos os sensores $( \pm 5 \%)$, visto que a tabela apresenta três valores maiores que $5 \%$.

Observa-se na tabela 2 que as médias da Uabc e da Up estão muito próximas $(8,5 \%)$, considerando que o erro médio dos instrumentos é de $( \pm 5 \%)$, pondera-se que as médias verificadas podem ser ainda mais semelhantes.
A umidade relativa verificada no abc mostrou-se menor que no abrigo oficial. Este fato ocorreu por fatores variáveis que podem estar associados inclusive à entrada de umidade no abrigo. Este erro, vale ressaltar, foi o maior erro verificado, pois atingiu $13,5 \%$. No entanto, apesar de ser o maior erro, o número encontrado é consideravelmente aceitável, visto as grandes variações de Umidade relativa que serão mostradas nas figuras abaixo.

A maior dispersão foi encontrada no abc, visto que sua amplitude atingiu $62,6 \%$, enquanto que a amplitude do Up foi de aproximados $52 \%$ (Uabc > Up). O desvio médio e o desvio padrão também apresentaram valores maiores no abc, 2,96\% e 2,92\%, respectivamente. Porém estes valores são relativamente semelhantes se comparados ao erro de ambos os instrumentos.

Nas figuras $07,08,09$ nota-se que o ponto máximo de umidade relativa foi atingido pelo Uabc em todos os horários, registrando $100 \%$ de umidade relativa do ar, valor este não atingido no Up, porém sabe-se que o erro médio de ambos os instrumentos é de $5 \%$, sendo a diferença entre máximas de 2,9\%.

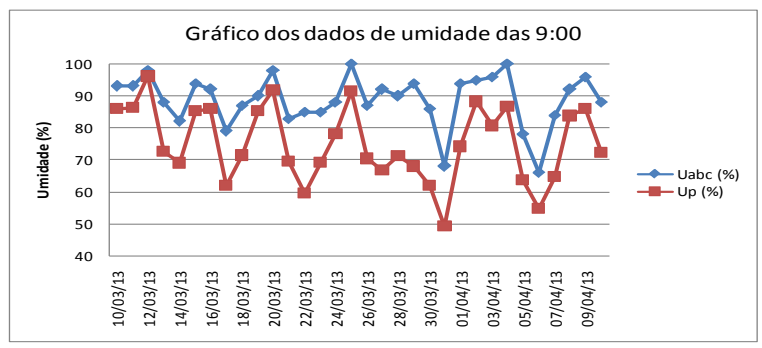

Figura 07 - Gráfico dos dados de Umidade das 9 horas

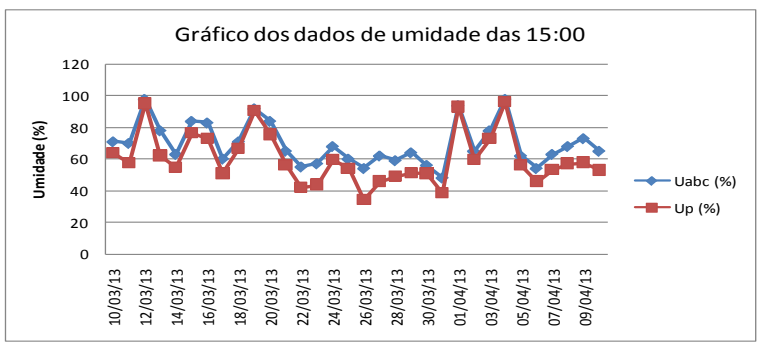

Figura 08 - Gráfico dos dados de Umidade das 15 horas

Tabela 2 - Séries de Umidades Relativas do Ar (\%) - parâmetros estatísticos elementares

\begin{tabular}{lrrr}
\hline \multicolumn{1}{c}{ Parâmetros } & Uabc (\%) & Up (\%) & Uabc-Up (\%) \\
\hline Média & 74,4 & 82,9 & $-8,5$ \\
Mínimo & 34,5 & 48,0 & $-13,5$ \\
Máximo & 97,1 & 100 & $-2,9$ \\
Amplitude & 62,6 & 52 & 10,6 \\
Desvio médio & 14,1 & 11,14 & 2,96 \\
Desvio Padrão & 16,4 & 13,48 & 2,92 \\
\hline
\end{tabular}




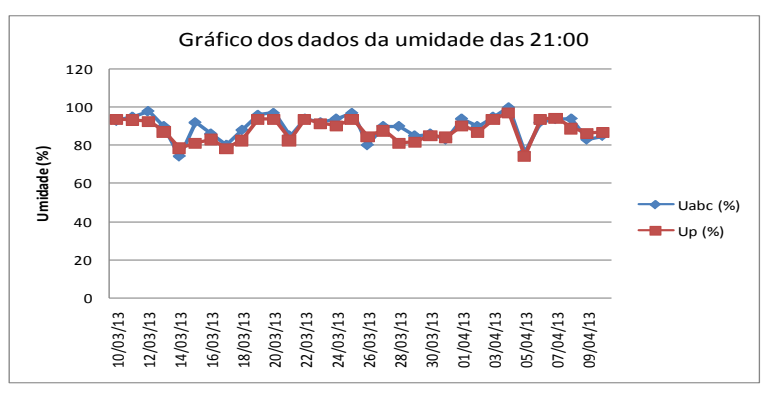

Figura 09 - Gráfico dos dados de Umidade das 21 horas

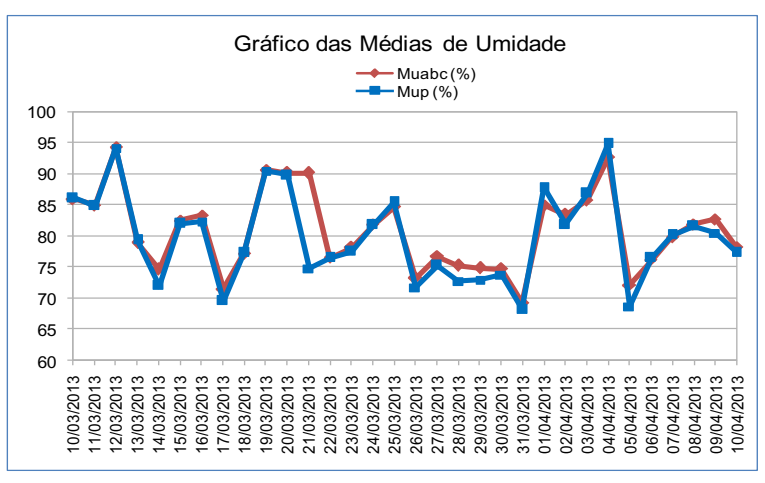

Figura 10 - Gráfico das médias de Umidade

\section{3 - Ponto de Orvalho}

Os dados do ponto de orvalho obtidos junto ao INMET são dados relativos à menor temperatura $\left({ }^{\circ} \mathrm{C}\right)$ do bulbo úmido. Devido aos valores serem coletados em três períodos diferentes do dia, o ponto de orvalho obtido possivelmente será do horário da manhã ou da noite $-09 \mathrm{~h}$ e $21 \mathrm{~h}$ respectivamente, pois a parte da tarde tende a possuir temperaturas mais elevadas, devido maior intensidade da radiação solar.

Os dados obtidos junto ao abrigo meteorológico foram de hora em hora, portanto realizou-se a média entre os três horários estudados, a fim de assemelhar-se com os dados oficiais do INMET.
Analisando a tabela 3 é possível verificar a que os dados médios são relativamente semelhantes, sendo a diferença de aproximadamente $2,3^{\circ} \mathrm{C}$. Destaca-se também a semelhança em temperaturas do ponto de orvalho mínima, a qual apresenta diferença de apenas $0,6^{\circ} \mathrm{C}$. Assim como a média, a máxima e a mínima, a amplitude também teve os valores de $\mathrm{PO} a b c>\mathrm{POp}$, onde neste caso obteve-se $1,6^{\circ} \mathrm{C}$ de diferença.

Por outro lado, porém com valores absolutamente semelhantes o Desvio médio e o Desvio padrão obtiveram valores minimamente maiores na estação oficial do INMET (POabc<Pop).

A figura 11 apresenta a comparação entre os dados do abc e os dados do INMET.

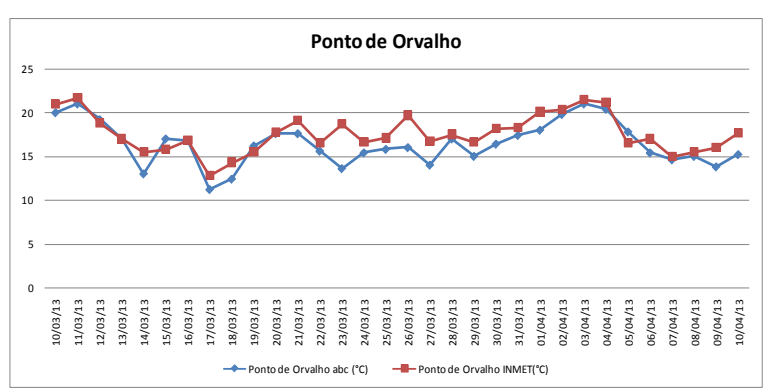

Figura 11 - Gráfico Ponto de Orvalho

Nota-se que apesar de iniciarem relativamente semelhantes, entre os dias 13/03/2013 e 16/03/2013 a linha representativa do INMET (marrom) tem uma queda chegando aos $13^{\circ} \mathrm{C}$, enquanto que a linha do abc (azul) fica próxima a $11^{\circ} \mathrm{C}$. Após este fato o ponto de orvalho do INMET volta a aumentar, e ao atingir $19^{\circ} \mathrm{C}$ a linha do abc atinge aproximadamente $17^{\circ} \mathrm{C}$, sendo o período de maior diferença entre ambos.

\section{4 - Pluviômetro}

O cálculo de ajuste do pluviômetro experimental com o oficial foi realizado com base na fórmula $V=\pi R^{2} h$, onde $\mathrm{V}$ é o volume coletado, $\pi$ é a constante, $\mathrm{R}^{2}$ é o raio do funil e h é a altura da chuva. A calibração do pluviômetro

Tabela 3 - Séries de Temp. do Ponto de Orvalho $\left({ }^{\circ} \mathrm{C}\right)$ - parâmetros estatísticos elementares

\begin{tabular}{|l|r|r|r|}
\hline \multicolumn{1}{|c|}{ Parâmetros } & POabc $\left({ }^{\circ} \mathbf{C}\right)$ & POp $\left({ }^{\circ} \mathbf{C}\right)$ & Poabc-POp $\left({ }^{\circ} \mathbf{C}\right)$ \\
\hline Média & 17,5 & 15,2 & 2,3 \\
\hline Mínimo & 11,8 & 11,2 & 0,6 \\
\hline Máximo & 23,2 & 21 & 2,2 \\
\hline Amplitude & 11,4 & 9,8 & 1,6 \\
\hline Desvio médio & 1,8 & 1,9 & $-0,1$ \\
\hline Desvio Padrão & 2,3 & 2,4 & $-0,1$ \\
\hline Desvio Padrão & 2,3 & 2,4 & $-0,1$ \\
\hline
\end{tabular}


foi analisada com o total de chuvas ocorridos entre os dias 09/03/2013 a 12/04/2013.

O volume coletado foi de 5,8 litros de chuvas totalizando em 280,6 $\mathrm{mm}$. Nesse sentido a calibração do pluviômetro com base na fórmula supracitada, baseando-se na metodologia de Milanesi (2007), resultou em $0,048 \mathrm{~mm}$ de chuva para cada $1 \mathrm{ml}$ de água coletada ou $48,4 \mathrm{~mm}$ de chuva para cada 1 litro de água coletada.

\section{Conclusões}

Ao analisar os dados obtidos verificou-se que o abc apresentou valores de temperatura diurna maiores que o da estação oficial. Este fato é percebido pelo aquecimento do aço pela radiação solar incidente durante o dia.

Já no período noturno pode-se verificar uma superior fidelidade do instrumento de baixo custo, pelo fato de este ser mais exposto a ventilação e consequentemente mais aberto que o abrigo utilizado pelo INMET.

Deste modo verificou-se que em locais onde existe estação meteorológica do INMET o abc apresenta-se como um aliado na coleta de variáveis, onde terá como resultado o valor médio do cálculo das duas estações.

No entanto, para análise do micro clima, ou em ambientes que não possuam estações meteorológicas do INMET, o abrigo meteorológico de baixo custo demonstra ser uma alternativa de fidelidade considerável, tornando-se assim, um método de abrigo de preço muito mais acessível do que os instrumentos disponíveis no mercado.

\section{Referências}

ARMANI, G. Variações da temperatura e umidade na bacia B do Núcleo Cunha - SP. In: SEMINÁRIO DE PESQUISA EM GEOGRAFIA FÍSICA, 1., São Paulo, 2003, São Paulo, SP. Anais..., São Paulo: Programa de Pós-Graduação em Geografia Física/FFCLH/USP, 2003. 821 p. p. $288-300$.

ARMANI, G.; GALVANI, E. Avaliação do desempenho de um abrigo meteorológico de baixo custo. Revista Brasileira de Agrometeorologia, Piracicaba-SP, v. 14, n.1, p. 116-122, 2006.

AZEVEDO, T. R. \& TARIFA, J. R. Mini abrigo meteorológico aspirado do Laboratório de Climatologia e Biogeografia e seu uso no estudo geográfico do clima. GEOUSP, São Paulo, n. 10, p. 165-174, 2001.

CASTELHANO, F.; ROSEGHINI, W. F. F. A utilização de policloreto de vinila (PVC) na construção de miniabrigos meteorológicos para a aplicação em campo. Revista Brasileira de Climatologia- Ano 07 - JUL/DEZ/2011, v. 9, p. $48-55$.
CUNHA, A. R. et al. Avaliação de um psicrômetro de termopar de baixo custo. Revista Brasileira de Agrometeorologia, Santa Maria, v. 9, n. 1, p. 17-22, 2001.

MILANESI, M. A.; GALVANI, E. Pluviômetro Experimental Para Localidades Remotas. In.: Climatologia Aplicada: Resgate aos Estudos de Caso. Galvani, E.; Lima, N. G. B. (Orgs.). Curitiba: Editora CRV, 2012. v. 1. 192 p. 1 ed.

MILANESI, M. A. Avaliação do efeito orográfico na pluviometria de vertentes opostas da Ilha de São Sebastião (Ilhabela-SP). Dissertação (Mestrado em Geografia Física). Universidade de São Paulo. São Paulo, 2007.

MONTEIRO, C. A. de F. Clima e Excepcionalismo Conjecturas sobre o desempenho da atmosfera como fenômeno geográfico. Editora da UFSC, Florianópolis, 1991.

WOLLMANN, C. A.; SARTORI, M. G. B. Frequência mensal e sazonal da participação de sistemas atmosféricos no verão do Rio Grande do Sul: análise sobre três casos típicos (1986/1987, 1997/1998 e 2004/2005). Revista Ciência e Natura, v. 31, p. 141-161, 2009b. 